\title{
ВЛИЯНИЕ ПОЛИОКСИДОНИЯ НА УРОВЕНЬ ПРОВОСПАЛИТЕЛЬНЫХ ЦИТОКИНОВ И ФУНКЦИОНАЛЬНУЮ АКТИВНОСТЬ НЕЙТРОФИЛОВ У БОЛЬНЫХ САХАРНЫМ ДИАБЕТОМ 2 ТИПА С ИНФЕКЦИЕЙ МОЧЕВЫХ ПУТЕЙ
}

\author{
Алибаева Г.Ф. ${ }^{1}$, Моругова Т.В. ${ }^{2}$, Насыртдинова А.Д. ${ }^{2}$, Чакрян С.А. ${ }^{3}$, Акчулпанов Н.Ф. ${ }^{4}$ \\ ${ }^{1}$ ГБУЗ РБ ГБ № 3, Стерлитамак, е-mail: Laim23@mail.ru; \\ ${ }^{2}$ ФГБОУ ВО «Башкирский государственный медииинский университет», Уфа, e-mail: tmorugova@yandex.ru; \\ ${ }^{3}$ ГБУЗ РБ Больница скорой медицинской помощи № 22, Уфыы; \\ ${ }^{4}$ ГБУЗ РБ КБ № 1, Стерлитамак, e-mail: NilI16@bk.ru
}

Исследовано влияние полиоксидония на уровень провоспалительных цитокинов и функциональную активность нейтрофилов у больных сахарным диабетом 2 типа (СД2) с инфекцией мочевых путей (ИМП). Было обследовано 158 человек: 31 - больные СД2, 35 - больные ИМП, 30 - больные СД2 с ИМП, 27 - больные СД2 с ИМП, получавшие полиоксидоний и 35 практически здоровых человек. Содержание

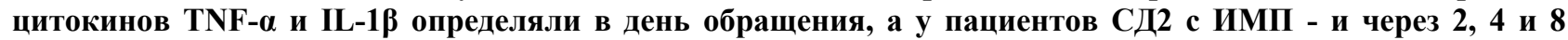
недель лечения. Фунциональное состояние нейтрофилов оценивали по интенсивности кислородзависимого метаболизма и фагоцитарной активности (ФАН). У больных СД2 был значительно

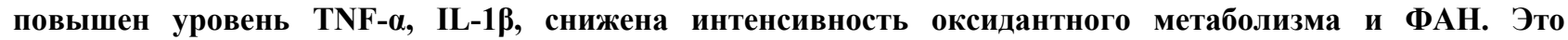
свидетельствует о наличии иммуновоспалительного процесса и подавлении защитной активности нейтрофилов. У больных СД2 с ИМП на фоне стандартной терапии сохранялся высокий уровень провоспалительных цитокинов и сниженная активность оксидантного метаболизма. Это свидетельствует об истощении резервных возможностей гранулоцитов, способствует затяжному течению ИМП и низкой эффективности проводимой терапии. Включение полиоксидония в терапию больных СД2

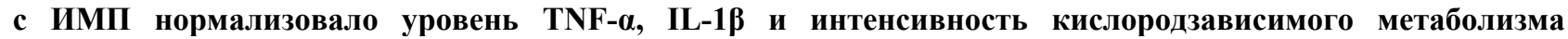
нейтрофилов. Таким образом, применение полиоксидония устраняет гиперпродукцию провоспалительных цитокинов, восстанавливает биоцидный потенциал и фагоцитарную активность нейтрофилов.

Ключевые слова: сахарный диабет, полиоксидоний, инфекции мочевых путей, цитокины, функциональная активность нейтрофилов.

\section{INFLUENCE OF POLYOXIDONIUM ON THE LEVEL OF PROVISOFLEX CYTOKINES AND THE FUNCTIONAL ACTIVITY OF NEUTROPHILS IN PATIENTS WITH TYPE 2 DIABETES MELLITUS WITH URINARY TRACT INFECTION}

\author{
Alibaeva G.F. ${ }^{1}$, Morugova T.V. ${ }^{2}$, Nasyrtdinova A.D. ${ }^{2}$, Chakryan S.A. ${ }^{3}$, Akchulpanov N.F. ${ }^{4}$ \\ ${ }^{1}$ State budgetary health care institution city hospital №3, Sterlitamak, e-mail: Laim23@mail.ru; \\ ${ }^{2}$ Bashkirian State Medical University, Ufa, e-mail: tmorugova@yandex.ru; \\ ${ }^{3}$ State budgetary health care institution city ambulance hospital №22, Ufa, \\ ${ }^{4}$ State budgetary health care institution city clinical hospital №1, Sterlitamak, e-mail: NilI16@bk.ru
}

The effect of polyoxidonium on the level of proinflammatory cytokines and the functional activity of neutrophils in patients with diabetes mellitus (DM 2) with urinary tract infection (UTI) was studied. 158 people were examined: 31 patients with DM 2, 35 patients with UTI, 30 patients with DM 2 with UTI, 27 patients with DM 2 with UTI who received polyoxidonium and 35 practically healthy people. The content of cytokines TNF- $\alpha$ and IL-1 $\beta$ was determined on the day of treatment, and in patients DM 2 with UTI - 2, 4 and 8 weeks of treatment.The functional state of neutrophils was assessed by the intensity of oxygen-dependent metabolism and their phagocytic activity (FAN). In patients with DM 2 the level of TNF- $\alpha$, IL-1 $\beta$ was significantly increased, the intensity of oxidative metabolism and FAN were decreased. This indicates the presence of an immunoinflammatory process and the suppression of the protective activity of neutrophil. In patients with DM 2 with UTI on the background of standard therapy maintained a high level of pro-inflammatory cytokines and a reduced activity of oxidative metabolism. This indicates the depletion of reserve capacity of granulocytes, contributes to the protracted course of UTI and low effectiveness of the therapy. The inclusion of polyoxidonium in the treatment of patients with diabetes with UTI normalized the level of TNF- $\alpha$, IL-1ß and the intensity of oxygen-dependent neutrophil metabolism. Thus, the use of polyoxidonium eliminates the hyperproduction of pro-inflammatory cytokines, restores the biocidal potential and the phagocytic activity of neutrophils.

Keywords: diabetes mellitus, polyocidonium, urinary tract infections, cytokines, functional activity of neutrophils. 
Течение сахарного диабета 2 типа (СД 2) часто сопровождается присоединением инфекций мочевых путей (ИМП), что существенно утяжеляет течение и ухудшает прогноз у больных СД 2.

С другой стороны, СД 2 - хронический иммуновоспалительный процесс, сопровождающийся развитием дисбаланса иммунной системы, что, в свою очередь, осложняет течение ИМП, снижает эффективность проводимой терапии [1-3]. Рост антибиотикорезистентности патогенной микрофлоры также снижает результативность терапии ИМП у больных СД 2.

В последние годы все большее внимание исследователей привлекает взаимодействие и взаимозависимость состояния иммунной и эндокринной систем. Особое место в этих процессах занимают цитокины, роль которых не ограничивается обеспечением адекватного иммунного ответа, активацией неспецифических защитных реакций и специфического иммунитета [4; 5]. Цитокины осуществляют интегративную функцию, обеспечивая ответ организма при попадании в него патогенного агента, путем вовлечения практически всех органов и систем [5].

В развитии СД 2, его осложнений, инсулинорезистентности, в прогрессировании воспаления при воздействии патогенных микроорганизмов существенная роль отводится провоспалительным цитокинам TNF- $\alpha$ и IL-1 $\beta[1 ; 5 ; 6]$.

TNF- $\alpha$ продуцируется макрофагами, T- и В-лимфоцитами, фибробластами и др. [4]. TNF- $\alpha$ повышает функциональную активность мононуклеарных фагоцитов, участвует в координации воспалительного ответа [4]. TNF- $\alpha$ является первичным медиатором воспаления, опосредует развитие системного воспаления, вовлекается в патогенез большинства иммунопатологических процессов [4; 5].

IL-1 $\beta$ - один из важнейших цитокинов острого воспалительного ответа. IL-1 $\beta$ увеличивает активность лимфоцитов, антителогенез, активность NK-клеток [7]. Путем повышения продукции других цитокинов, в частности IL-8, IL-1 $\beta$ стимулирует активность нейтрофильных гранулоцитов. Также IL-1 $\beta$ оказывает прокоагулянтное действие, нарушает микроциркуляцию [6]. В условиях гипергликемии повышается продукция IL-1 $\beta$, который способен запускать апоптоз $\beta$-клеток [8].

В процессе лечения необходимо контролировать уровень цитокинов, так как их повышение в острую фазу воспалительного ответа носит защитный характер, в то время как длительное и бесконтрольное повышение уровня цитокинов может приводить к необратимым нарушениям гомеостаза [4; 5].

Ключевую роль в развитии и течении воспалительного процесса играют нейтрофилы - клетки, участвующие в противоинфекционной защите организма, участвующие в 
процессах иммуногенеза, противоопухолевой резистентности, а также в нейроэндокринной регуляции адаптационных реакций организма [9].

Нейтрофилы - клетки, крайне чутко реагирующие на изменение гомеостаза, что дает возможность использовать их для оценки состояния всей иммунной системы [9].

Длительность течения и низкая результативность стандартной терапии ИМП у больных СД 2 в условиях дисбаланса иммунной системы обосновывают необходимость использования иммуномодуляторов в комплексной терапии ИМП у больных СД 2.

В качестве иммунокорректора нами был выбран отечественный препарат полиоксидоний (Пд) - физиологически активное высокомолекулярное соединение, которое относится к классу водорастворимых производных гетероцепных алифатических полиаминов, является сополимером N-окси 1,4-этиленпиперазина и (N-карбоксиэтил)-1,4этиленпиперазиний бромида.

Полиоксидоний оказывает стимулирующее влияние на факторы неспецифической защиты организма, гуморальный и клеточный иммунитет [10-12]. Важной особенностью полиоксидония является его активирующее воздействие на противоинфекционную резистентность организма [10]. Препарат обладает также антиокислительным, мембраностабилизирующим и дезинтоксикационным действием [10]. Полиоксидоний стимулирует антимикробную активность гранулоцитов, цитотоксичность NK-клеток, антителогенез [12]. Рядом авторов показано, что включение полиоксидония в комплексную терапию острых и хронических инфекций существенно повышает эффективность лечения $[11 ; 12]$.

В связи с этим целью исследования явилась оценка влияния полиоксидония на уровень провоспалительных цитокинов и функциональное состояние нейтрофилов у больных СД 2 с ИМП.

\section{Материалы и методы исследования}

В исследование вошло 158 человек, которые были разделены на 5 групп: 1 группа «здоровые» (35 человек), 2 группа - «СД 2» (31 человек), 3 группа - «ИМП» (35 человек), 4 группа - «СД 2 + ИМП», 5 группа - «СД 2 + ИМП + Пд» (27 человек).

Пациенты, больные СД 2, не отличались по полу, возрасту, индексу массы тела (ИМТ) (таблица). Пациенты получали стандартную сахароснижающую терапию.

Больные ИМП получали лечение в соответствии с российскими клиническими рекомендациями [13]. Группа «СД 2+ИМП+Пд» дополнительно получала полиоксидоний в дозе 6 мг 1 раз в сутки в течение 10 дней (НПО «Петровакс Фарм» ООО, Россия). 
Характеристика обследованных групп

\begin{tabular}{|l|l|c|c|c|c|c|}
\hline \multirow{2}{*}{$\begin{array}{l}\text { № } \\
\text { № }\end{array}$} & \multicolumn{1}{|c|}{ Группы } & \multirow{2}{*}{$\begin{array}{c}\text { Кол- } \\
\text { во }\end{array}$} & \multicolumn{2}{|c|}{ Пол } & \multirow{2}{*}{ Возраст (лет) } & \multirow{2}{*}{ ИМТ } \\
\hline 1. & Здоровые & 35 & $11(31,43 \%)$ & $24(68,57 \%)$ & $43,0[39,0-50,0]$ & $24,80[22,50-25,80]$ \\
\hline 2. & СД 2 & 31 & $14(45,16 \%)$ & $17(54,84 \%)$ & $60,0[53,0-63,0]$ & $29,60[27,40-34,90]$ \\
\hline 3. & ИМП & 35 & $15(42,86 \%)$ & $20(57,14 \%)$ & $56,0[47,0-63,0]$ & $27,05[23,50-28,60]$ \\
\hline 4. & СД 2+ ИМП & 30 & $10(33,33 \%)$ & $20(66,67 \%)$ & $\begin{array}{c}56,50[50,0- \\
61,0]\end{array}$ & $32,0[28,60-33,50]$ \\
\hline 5. & $\begin{array}{l}\text { СД 2 + ИМП } \\
+ \text { Пд }\end{array}$ & 27 & $3(11,11 \%)$ & $24(88,89 \%)$ & $61,0[50,0-67,0]$ & $30,00[26,70-32,90]$ \\
\hline
\end{tabular}

Диагноз сахарного диабета, типа и степени тяжести заболевания устанавливался согласно «Алгоритмам специализированной медицинской помощи больным сахарным диабетом» (8-й выпуск, 2017 г.). Критериями включения в исследование явились: возраст от 18 лет, предварительно подтвержденный диагноз СД 2, удовлетворительный контроль СД 2 (гликемия натощак $<7,0$ ммоль/л; гликемия постпрандиальная $<19,0$ ммоль/л; НbА1<7\%); верифицированный диагноз ИМП (пиелонефрита), письменное информированное согласие пациента на проведение исследований и соблюдение этических принципов.

У всех обследованных определяли уровень цитокинов: фактор некроза опухолей-

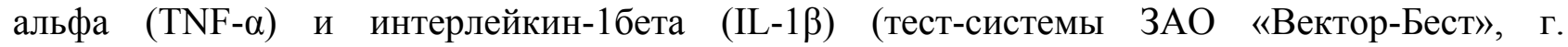
Новосибирск) в день обращения. У пациентов групп «СД $2+$ ИМП» и «СД $2+$ ИМП + Пд» содержание цитокинов определяли также через 2, 4 и 8 недель лечения (на 2, 3 и 4-й визиты). Также исследовали интенсивность кислородзависимого метаболизма (спонтанный (НСТсп) и индуцированный (НСТинд) НСТ-тест) и фагоцитарную активность нейтрофилов (ФАН) [14].

Статистическая обработка данных проводилась с использованием методов вариационной статистики [15], пакета прикладных программ Statistica 8.0 на персональном компьютере. Показатели представлены в виде медианы (Ме) и квартилей (Р25\%; Р75\%). Дисперсионный анализ проводили с помощью Н-критерия Краскела-Уоллиса, для множественных сравнений использован Q-критерий Дана. Результаты считались статистически значимыми при $\mathrm{p}<0,05$.

\section{Результаты исследования и их обсуждение}

В группе «СД 2» отмечено увеличение уровня TNF- $\alpha$ в 2,3 раза по сравнению с группой здоровых лиц $(\mathrm{p}=0,000001)$ и в 1,5 раза - с «ИМП» $(\mathrm{p}=0,02399)$ (рис. 1). Также отмечено увеличение IL-1 $\beta$ в 2 раза по сравнению со здоровыми ( $\mathrm{p} \leq 0,00001)$ и почти на $40 \%$ по сравнению с группой «ИМП» (p=0,00189) (рис. 2). 


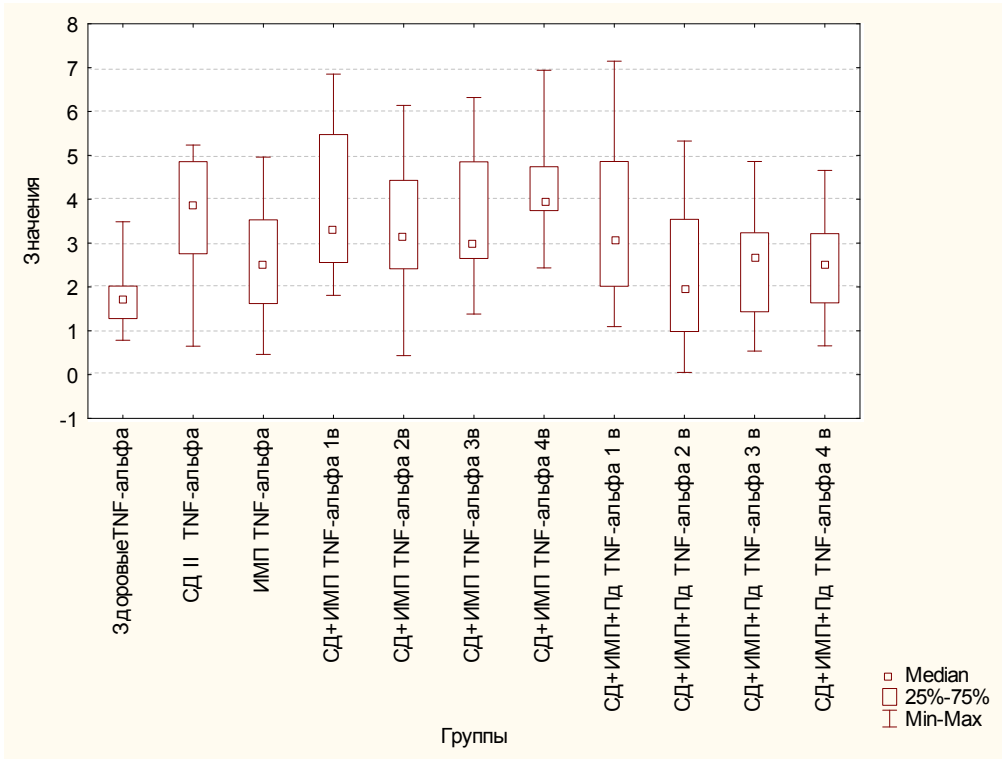

Рис. 1. Уровень TNF-а (пг/мл) у здоровых, больных сахарнымм диабетом 2 типа, больных инфекцией мочевых путей, больных сахарным диабетом 2 типа и инфекцией мочевых путей, больных сахарным диабетом 2 типа и инфекцией мочевых путей и получающих полиоксидоний

Также у пациентов этой группы было установлено снижение ФАН в 5 раз по сравнению с группой «здоровые» $(\mathrm{p} \leq 0,00001)$ и в 3,2 раза - с группой «ИМП» $(\mathrm{p} \leq 0,00001)$ (рис. 3).

Наблюдалось 2-кратное подавление оксидантного метаболизма нейтрофилов в спонтанном и индуцированном НСТ-тесте по сравнению как с группой здоровых лиц $(\mathrm{p} \leq 0,00001$ и $\mathrm{p} \leq 0,00001$ соответственно), так и с группой «ИМП» $(\mathrm{p} \leq 0,00001$ и $\mathrm{p} \leq 0,00001$ соответственно). Это свидетельствует о том, что СД 2 является хроническим иммуновоспалительным процессом с формированием глубокого дисбаланса иммунной системы и снижением противомикробной защиты и согласуется с данными других авторов $[3]$.

При присоединении ИМП у больных СД 2 сохранялся высокий уровень провоспалительных цитокинов без тенденции к снижению к окончанию лечения. Так, уровень TNF- $\alpha$ к 4-му визиту ( $p=0,000030)$, а IL-1 $\beta$ - на протяжении всего периода наблюдения были достоверно выше, чем у больных ИМП в острый период заболевания ( $\mathrm{p}=0,00217, \mathrm{p}=0,00145, \mathrm{p}=0,00017$ соответственно на 2, 3 и 4-й визиты) (рис. 1, 2). 


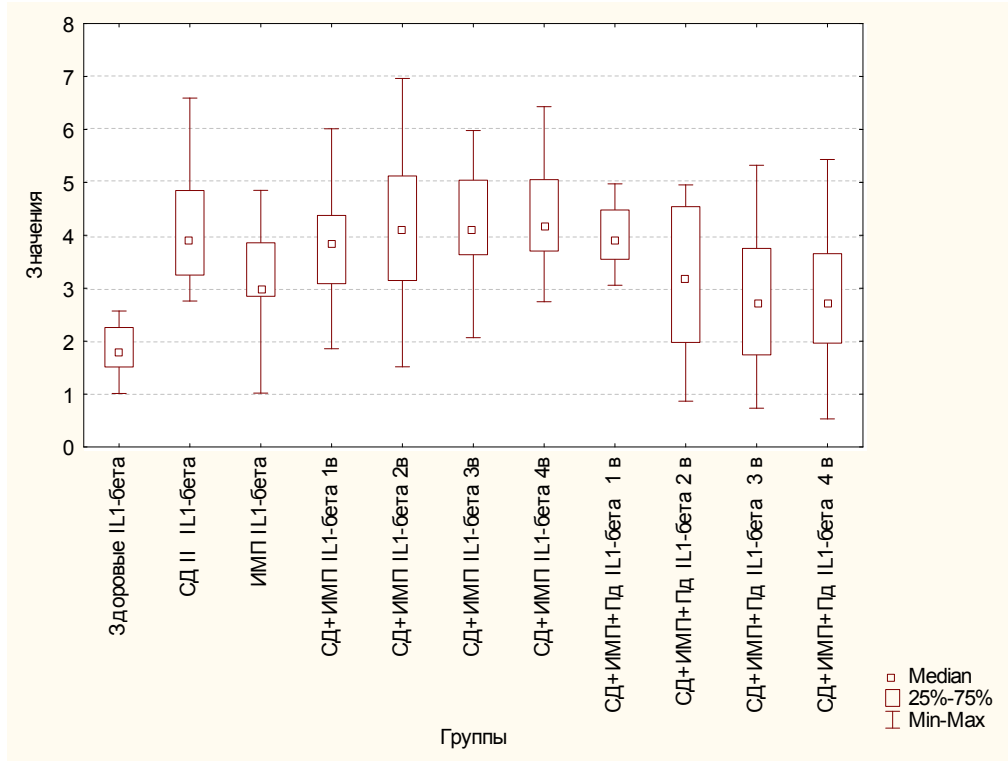

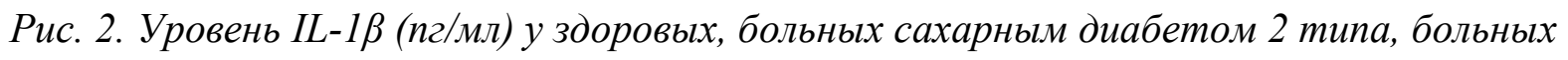
инфекиией мочевых путей, больных сахарным диабетом 2 типа и инфекцией мочевых путей, больных сахарным диабетом 2 типа и инфекиией мочевых путей и получающих полиоксидоний

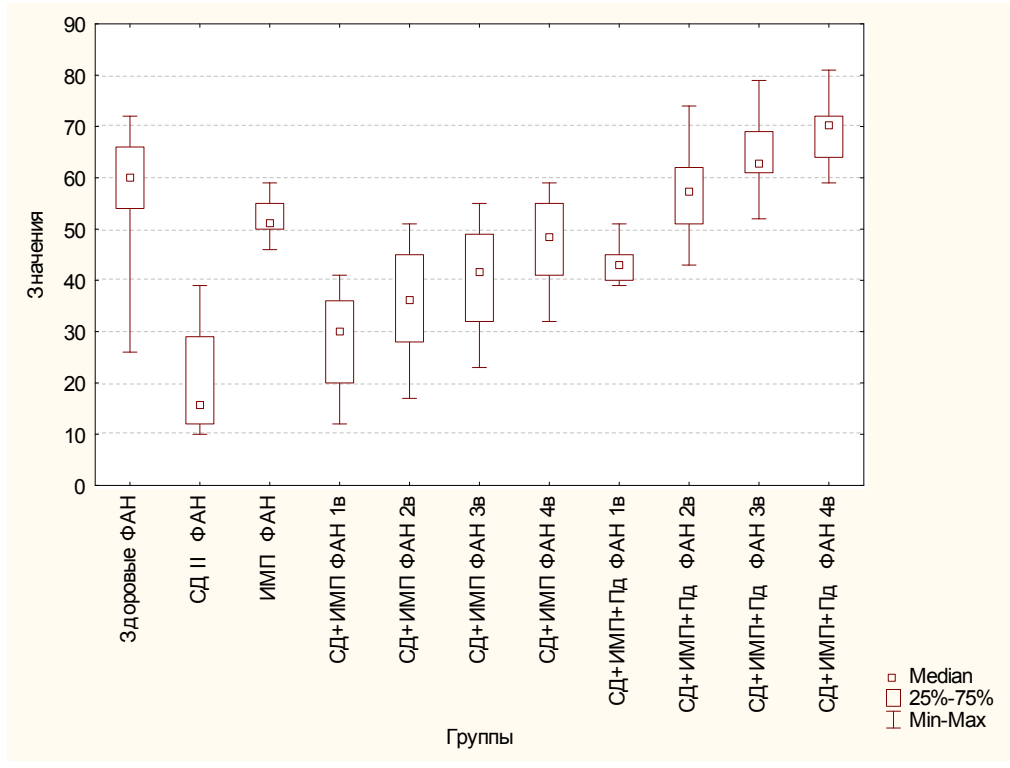

Рис. 3. Фагоцитарная активность нейтрофилов у здоровых, больных сахарным диабетом 2 типа, больных инфекиией мочевых путей, больных сахарным диабетом 2 типа и инфекичией мочевых путей, больных сахарным диабетом 2 типа и инфекцией мочевых путей и получающих полиоксидоний 
В группе «СД 2+ИМП» выявлено снижение ФАН на $50 \%$ по сравнению с группой здоровых лиц $(\mathrm{p} \leq 0,00001)$ и на $30 \%$ - с группой «ИМП» $(\mathrm{p} \leq 0,00001)$, однако на 30\% выше, чем в группе «СД 2», что можно объяснить антигенной стимуляцией (рис. 3). К 4-му визиту уровень ФАН повышался до уровня группы «ИМП» (в острый период) и составил 80,83\% (по отношению к уровню здоровых лиц). Аналогично показатели кислородзависимого метаболизма нейтрофилов в спонтанном НСТ-тесте не отличались от таковых в группе «СД 2» и лишь к 4-му визиту повышались до 84,44\% (по сравнению с группой здоровых лиц) и были сопоставимы с таковыми у больных «ИМП» в острый период заболевания. В то же время в условиях индукции показатели активности нейтрофилов оставались крайне низкими и даже на 4-й визит составили лишь 69,79\% (от уровня здоровых лиц), не достигая уровня больных группы «ИМП» в острый период. Полученные данные могут свидетельствовать об истощении резервных возможностей гранулоцитов, отсутствии адекватного ответа на воздействие патогенов, что, безусловно, способствует затяжному течению ИМП и низкой эффективности проводимой терапии.

Добавление Пд в комплексную терапию больных СД 2 с ИМП обеспечило статистически значимое снижение уровня TNF- $\alpha$ начиная со 2-го визита, и к 4-му визиту содержание TNF- $\alpha$ было ниже на 35\% по сравнению с группой больных СД 2 (p=0,00472, $\mathrm{p}=0,02004, \mathrm{p}=0,04111$ на 2, 3 и 4-й визиты соответственно) и «СД 2+ИМП» (p=0,00007, $\mathrm{p}=0,00045, \mathrm{p}=0,00097$ на 2, 3 и 4-й визиты соответственно). На 1-м визите уровень TNF- $\alpha$ не отличался от такового от группы «ИМП» $(\mathrm{p}=0,14153)$ (рис. 1).

Аналогично применение Пд обеспечило достоверное снижение уровня IL-1 $\beta$ на 3-й и 4-й визиты по сравнению с больными СД 2 ( $\mathrm{p}=0,01382$ и $\mathrm{p}=0,00666$ соответственно) и не отличалось от уровня у здоровых лиц ( $\mathrm{p}=0,18964$ и $\mathrm{p}=0,34263$ соответственно) (рис. 2). Полученные данные свидетельствуют о выраженном противовоспалительном эффекте Пд и коррекции им иммунного дисбаланса в группе «СД $2+$ ИМП».

Применение Пд у больных в группе «СД $2+$ ИМП» обеспечило статистически

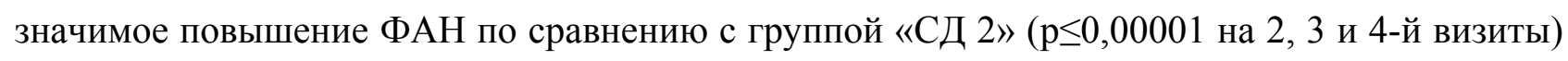
и группой «СД 2+ИМП» $(\mathrm{p}=0,00001, \mathrm{p} \leq 0,00001, \mathrm{p}=0,00001$ на 2, 3 и 4-й визиты соответственно) (рис. 3). Уже со 2-го визита ФАН в группе «СД 2+ИМП+Пд» не отличалась от таковой в группе здоровых лиц (p=1,0000 на 2, 3 и 4-й визиты) (рис. 3).

Применение Пд не изменяло показателей интенсивности оксидантного метаболизма нейтрофилов в спонтанном НСТ-тесте, что можно объяснить антиоксидантной активностью Пд [12]. В то же время в условиях индукции было отмечено статистически значимое повышение активности нейтрофилов к 4-му визиту (на $30 \%$ по сравнению с группой «СД 2», $\mathrm{p}=0,02056)$, что не отличалось от показателей в группе «здоровые» $(\mathrm{p}=0,71529)$. Полученные 
результаты могут свидетельствовать о повышении Пд биоцидности нейтрофилов у больных СД 2 с ИМП, что способствует более эффективной элиминации возбудителей инфекции и повышению результативности проводимой терапии. Это согласуется с тем, что Пд активирует фагоцитирующие клетки, восстанавливает иммунные реакции при вторичных иммунодефицитных состояниях [10;12].

Таким образом, применение Пд у больных СД 2 с ИМП обеспечило достоверное снижение уровня провоспалительных цитокинов (TNF- $\alpha$ и IL1 $\beta$ ), повышение фагоцитарной активности и интенсивности кислородзависимого метаболизма нейтрофилов.

Результаты проведенного исследования свидетельствуют о том, что включение Пд в лечение больных СД 2 с ИМП оказывает позитивное влияние на дисбаланс цитокинов и функциональную активность нейтрофильных гранулоцитов.

\section{Выводы}

1. В группе «СД 2» выявлено значительное повышение уровня цитокинов TNF- $\alpha$ и IL-1 $\beta$, подавление фагоцитарной и антимикробной активности нейтрофилов.

2. В группе «СД $2+$ ИМП» при проведении стандартной терапии уровень цитокинов TNF- $\alpha$ и IL-1 $\beta$ сохраняется на высоком уровне, также отмечается снижение поглотительной и микробицидной способности нейтрофильных гранулоцитов.

3. Включение полиоксидония в комплексную терапию в группе «СД $2+$ ИМП + Пд» снижает уровень цитокинов TNF- $\alpha$ и IL-1 $\beta$, восстанавливает фагоцитарную активность и биоцидный потенциал нейтрофилов.

\section{Список литературы}

1. Аметов С.А. Как снизить риск развития осложнений у пациентов с СД 2 типа? Выбор терапевтической тактики // Эффективная фармакотерапия. 2017. №9. С. 46-48.

2. Donath M., Shoelson S. Type 2 diabetes as an inflammatory disease. Nat. Rev. Immunol. 2011. Vol. 11. P. 98-107.

3. Grant R., Dixit W. Mechanisms of disease: inflammasome activation and the development of type 2 diabetes. Front. Immunol. 2013. Vol. 4. P. 50.

4. Кетлинский С.А., Симбирцев А.С. Цитокины. СПб: ООО «Издательство Фолиант», 2008. $552 \mathrm{c}$.

5. Симбирцев А.С. Цитокины в патогенезе инфекционных и неинфекционных заболеваний человека // Медицинский академический журнал. 2013. Т.13. №3. С.18-41.

6. Бабаева А.Р., Тарасов А.А., Безбородова Е.А., Захарьина О.А. Концепция системного воспаления в патогенезе диабетической ангиопатии // Вестник ВолГМУ. 2010. Выпуск 1 (33). 
C. 3-8.

7. Dinarello C. Immunological and Inflammatory Functions of the Interleukin-1 Family. Ann. Rev. Imm. 2009. Vol. 27. P. 519-550.

8. Maedler K., Sergeev P., Ris F., Oberholzer J., Joller-Jemelka H.I., Spinas G.A., Kaiser N., Halban P.A., Donath M.Y. Glucose-induced beta-cell production of IL-1beta contributes to glucotoxicity in human pancreatic islets. J. Clin. Invest. 2002. Vol. 110. P. 851-860.

9. Борздов А.А., Логвиненко О.В., Борздова И.Ю., Ефременко В.И. Функциональные особенности нейтрофильных гранулоцитов при введении интактных липосом // Медицинский вестник Северного Кавказа. 2009. Т. 15. №3. С. 53-55.

10. Петров Р.В., Хаитов Р.М., Некрасов А.В., Аттаулаханов Р.И., Пучкова Н.Г., Иванова А.С., Пинегин Б.В., Хаумидуллина К.Ф., Дамбаева С.В., Климова С.В. Полиоксидоний: механизм действия и клиническое применение // Медицинская иммунология. 2000. № 3. С. 271-278.

11. Фомичев Е.В., Поройский С.В., Кирпичников М.В. Современная концепция лечения гнойно-воспалительных заболеваний лица и шеи у больных сахарным диабетом // Волгоградский научно-медицинский журнал. 2014. №3. С.35-40.

12. Зарубина И.В., Мокренко Е.В., Болехан А.В. Противовоспалительная и иммуномодулирующая активность метапрота, трекрезана и полиоксидония и их комбинаций при экспериментальном бронхолегочном воспалении у крыс // Вестник Смоленской государственной медицинской академии. 2016. Т. 15. №1. С. 5-13.

13. Урология. Российские клинические рекомендации / ред. Ю. Г. Аляев, П. В. Глыбочко, Д. Ю. Пушкарь. М.: ГЭОТАР-Медиа, 2016. 496 с.

14. Afzal M.M., Jeshtadi A., Mohmmed A.K. Study of neutrophilic function by nitroblue tetrazolium test in septicemias and immune deficiency diseases. Int. J. Res. Health Sci. 2014. no.2(2). P.581-90.

15. Гареев Е.М. Основы математико-статистической обработки медико-биологической информации. Уфа: Изд-во ГОУ ВПО «Башгосмедуниверситет Роздрава», 2009. 346 с. 